\title{
Das Kursbuch 2016 der Dentaurum-Gruppe
}

\section{Jahre CDC - Centrum Dentale Communikation}

Seit der Gründung im Jahre 1996 hat das CDC, Centrum Dentale Communikation, unter den verschiedenen Fortbildungszentren im Dentalbereich eine führende Rolle eingenommen. Sei es durch das breite Angebot an Kursen und Veranstaltungen für Zahnärzte, Kieferorthopäden, Zahntechniker und zahnmedizinische Fachangestellte oder durch die überzeugende Infrastruktur und die transparente Architektur des CDC.

Doch die Dentaurum-Gruppe steht nicht erst seit Eröffnung des CDC für qualitativ hochwertige Kurse und Veranstaltungen. Sie verfügt vielmehr über eine langjährige Tradition und Erfahrung bei der Organisation von Kursen.

Das erste Kursprogramm nach Gründung des CDC im Jahre 1996 umfasste 30 Kurse in den Bereichen Modellguss, Kronenund Brücken, Titan- und Lasertechnik, Orthodontie, Kieferorthopädische Zahntechnik, Implantologie, Keramik und Praxis- und Labormanagement. Mittlerweile werden mehr als 130 attraktive Kurse und Veranstaltungen von über 100 Referenten deutschlandweit $\mathrm{zu}$ den unterschiedlichsten Themen angeboten.

Implantologie: große Vielfalt Das Veranstaltungshighlight in der Implantologie im April 2016 ist die International Dental Conference in Berlin. Ein engagiertes und erfahrenes Referenten-Team führt die Teilnehmer aus aller Welt drei Tage lang durch praxisbezogene Workshops, Diskussionen und aktuelle Vorträge. Weiterhin finden an renommierten deutschen Universitäten die stets gefragten Implantationskurse an Human- präparaten, die Fachtage Implantologie und die Kursreihen „Implantology step by step“ statt.

Noch mehr Kieferorthopädie In der Orthodontie wurde das Kursangebot in den letzten Jahren deutlich erweitert. 2016 neu dabei sind die innovative Behandlung von Klasse II Gebissanomalien mit amda $\odot$ (advanced molar distaliziation appliance) und Veranstaltungen zur digitalen Kieferorthopädie. Erstmalig bietet die Dentaurum-Gruppe Fortbildungen in Zusammenarbeit mit der Haranni Academie in Herne an.

\section{Kieferorthopädische \\ Zahntechnik}

In der kieferorthopädischen Zahntechnik ist die Auswahl groß - das Programm reicht von Kursen für Einsteiger bis hin zu Spezialkursen zu unterschiedlichen Apparaturen. Bekannte Referenten geben ihr umfangreiches Fachwissen an die Teilnehmer weiter.

\section{Zahntechnik: neue Kurse}

In der Zahntechnik wurden die beliebten ModellgussKurse durch Kurse zur Teleskop- und Lasertechnik sowie vielfältige Keramik-Kurse ergänzt. Von einem erstklassigen Experten-Team werden das Handling mit dem ceraMotion ${ }^{\circledR}$ Keramiksystem demonstriert und die verschiedenen Anwendungsmöglichkeiten dargestellt.

Als besonderes Highlight im CDC-Jubiläumsjahr profitieren die Kursteilnehmer beim Kurs des Monats von $20 \%$ Jubiläumsrabatt. Um welchen Kurs es sich monatlich handelt, erfährt man direkt im Kursbuch 2016 oder unter www.dentaurum.de.

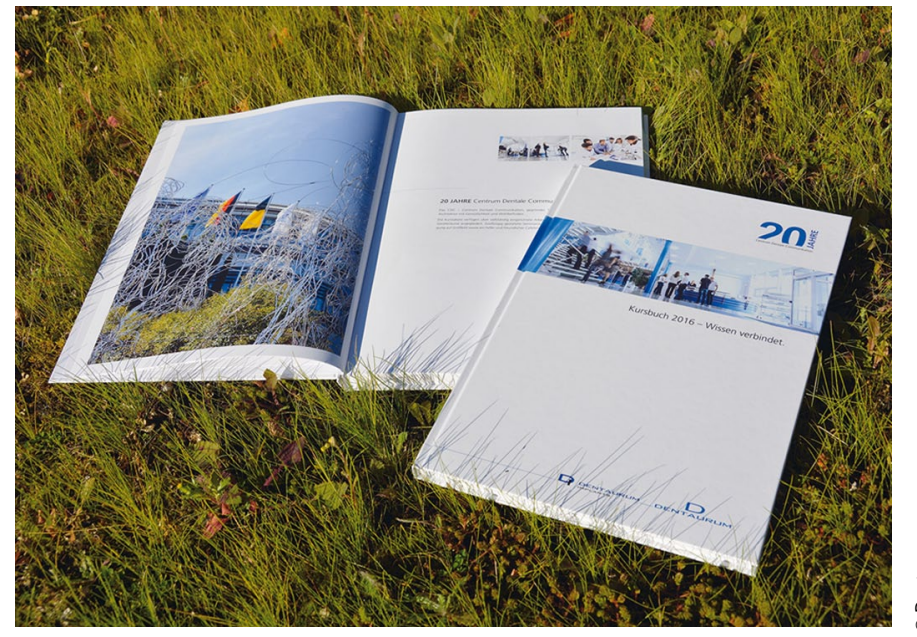

$\Delta$ Das Dentaurum Kursbuch 2016: ein attraktives Veranstaltungsangebot erwartet Sie.

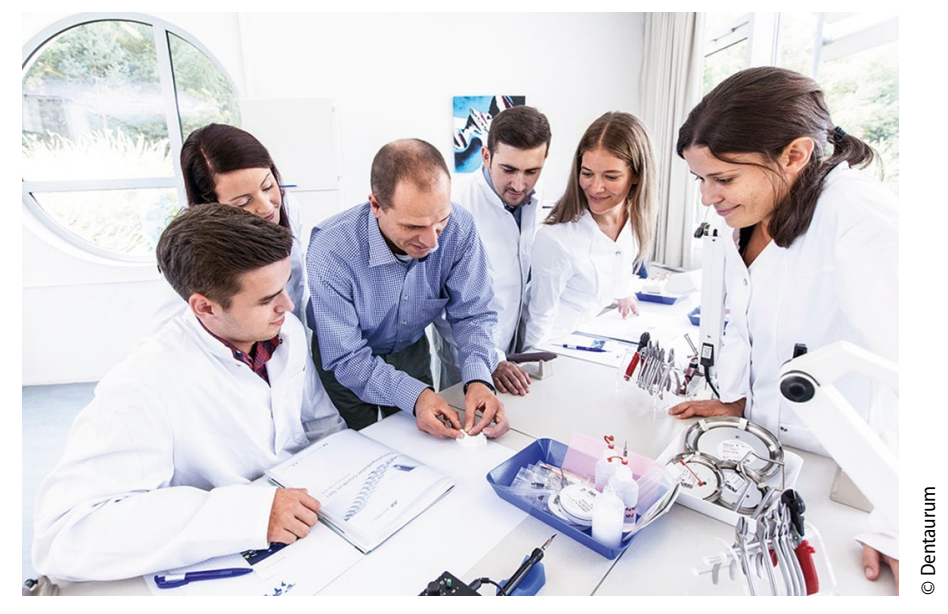

$\Delta$ Kleine Gruppen machen das Lernen und Auffrischen leicht.

Das Kursbuch 2016 kann angefordert werden bei:

DENTAURUM GmbH \& Co. KG Centrum Dentale Communikation Turnstr. 31, D-75228 Ispringen CDC-Team: Tel. +49(0)7231/803-470, Fax: +49 (0)7231/803-409

E-Mail: kurse@dentaurum.de Web: www.dentaurum.com 